Article

\title{
Temperature Differentially Affects Gene Expression in Antarctic Thraustochytrid Oblongichytrium sp. RT2316-13
}

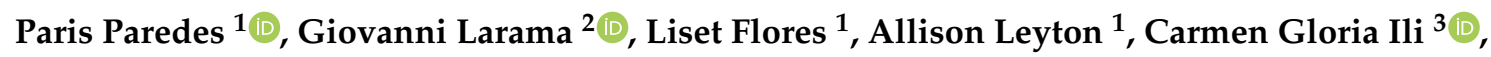
Juan A. Asenjo ${ }^{4}$, Yusuf Chisti ${ }^{5}\left(\mathbb{D}\right.$ and Carolina Shene ${ }^{1, * \mathbb{C}}$

1 Department of Chemical Engineering, Center of Food Biotechnology and Bioseparations, BIOREN, and Centre of Biotechnology and Bioengineering (CeBiB), Universidad de La Frontera, Av. Francisco Salazar 01145, Temuco 4780000, Chile; p.paredes03@ufromail.cl (P.P.);

liset.flores@ufrontera.cl (L.F.); allison.leyton@ufrontera.cl (A.L.)

2 Centro de Modelación y Computación Científica, Universidad de La Frontera, Av. Francisco Salazar 01145, Temuco 4780000, Chile; giovanni.larama@ufrontera.cl

3 Centro de Excelencia en Medicina Traslacional—Scientific and Technological Bioresource Nucleus (CEMT-BIOREN), Universidad de La Frontera, Av. Alemania 0478, Temuco 4810296, Chile; carmen.ili@ufrontera.cl

4 Centre for Biotechnology and Bioengineering (CeBiB), Department of Chemical Engineering and Biotechnology, Universidad de Chile, Beauchef 851, Santiago 8370459, Chile; juasenjo@ing.uchile.cl

5 School of Engineering, Massey University, Private Bag 11 222, Palmerston North 4442, New Zealand; Y.Chisti@massey.ac.nz

* Correspondence: Carolina.shene@ufrontera.cl; Tel.: +56-45-232-5491

Received: 22 October 2020; Accepted: 11 November 2020; Published: 18 November 2020

\begin{abstract}
Oblongichytrium RT2316-13 synthesizes lipids rich in eicosapentaenoic acid (EPA) and docosahexaenoic acid (DHA). The content of these fatty acids in the total lipids depended on growth temperature. Sequencing technology was used in this work to examine the thraustochytrid's response to a decrease in growth temperature from $15{ }^{\circ} \mathrm{C}$ to $5{ }^{\circ} \mathrm{C}$. Around $4 \%$ (2944) of the genes were differentially expressed (DE) and only a few of the DE genes (533 upregulated; 206 downregulated) had significant matches to those in the SwissProt database. Most of the annotated DE genes were related to cell membrane composition (fatty acids, sterols, phosphatidylinositol), the membrane enzymes linked to cell energetics, and membrane structure (cytoskeletal proteins and enzymes). In RT2316-13, the synthesis of long-chain polyunsaturated fatty acids occurred through w3- and $\omega 6$-pathways. Enzymes of the alternative pathways ( $\Delta 8$-desaturase and $\Delta 9$-elongase) were also expressed. The upregulation of the genes coding for a $\Delta 5$-desaturase and a $\Delta 5$-elongase involved in the synthesis of EPA and DHA, explained the enrichment of total lipid with these two long-chain fatty acids at the low temperature. This molecular response has the potential to be used for producing microbial lipids with a fatty acids profile similar to that of fish oils.
\end{abstract}

Keywords: gene expression analysis; lipid metabolism; Oblongichytrium sp.; polyunsaturated fatty acid synthesis; thraustochytrids

\section{Introduction}

Marine thraustochytrids (heterotrophic biflagellate single-celled eukaryotes) are good producers of lipids rich in docosahexaenoic acid (DHA, C22:6 $6^{\Delta 4,7,10,13,16,19}$ ). In fact, commercial production of DHA for human food relies on thraustochytrids such as Schizochytrium sp. and Ulkenia sp. [1,2]. Although most of the studied thraustochytrids produce DHA as the main long chain polyunsaturated fatty acid 
(LCPUFA), other strains in this group also synthesize eicosapentaenoic acid (EPA, C20:5 ${ }^{\Delta 5,8,11,14,17}$ ). Dietary consumption of lipids rich in EPA and DHA has many beneficial effects on health [3]. At present, marine fish are the main source of oils rich in EPA and DHA. Many microorganisms synthesize EPA and DHA and may in the future contribute to biotechnological production of lipids similar to fish oil.

Cold-adapted microbes commonly have an elevated level of unsaturated fatty acids in their cell membranes [4-6] possibly because lipid bilayers containing unsaturated fatty acids retain fluidity at relatively low temperatures [7] compared to bilayers with a preponderance of saturated fatty acids. Elevated levels of DHA and/or EPA have been observed in some marine species grown at low temperature [8-10], but this is not always so [11] and there is some uncertainty about the exact influence of EPA and DHA on the properties of lipid bilayer membranes. For example, although a reduced culture temperature elevated the EPA level, this fatty acid was claimed to not influence the bulk bilayer fluidity in the gram-negative bacterium Shewanella livingstonensis Ac10 [6]. Similarly, in the gram-negative deep-sea bacterium Photobacterium profundum SS9, EPA levels did increase in low temperature cultivation, but this was concluded to be nonessential for low-temperature growth [4]. Levels of EPA and DHA in thraustochytrid total lipids depend on the culture conditions [12,13], including temperature of cultivation [14].

The thraustochytrid Oblongichytrium RT2316-13 isolated from Antarctic seawaters [15] was the focus of the present study. This microorganism grew at temperatures ranging from 2 to $15^{\circ} \mathrm{C}$ and, therefore, could be classified as a psychrophile [16]. RT2316-13 was shown to produce DHA and EPA [14]. Cultures grown at 5 and $15^{\circ} \mathrm{C}$ attained the same final biomass concentration, although at $15{ }^{\circ} \mathrm{C}$ the specific growth rate was 4.6 -fold greater than at $5^{\circ} \mathrm{C}\left(0.032 \mathrm{~h}^{-1}\right)$ [14]. Although the biomass grown at $5{ }^{\circ} \mathrm{C}$ had significantly fewer lipids than the biomass grown at $15^{\circ} \mathrm{C}$, the proportions of DHA and EPA in the total lipids were higher in the cells grown at the lower temperature [14]. Therefore, the growth temperature was postulated to differentially affect the expression of at least some of the genes relating to the metabolism of fatty acids. To better understand the temperature-dependent metabolism of LCPUFA in Oblongichytrium RT2316-13, the present work examined the differential expression of genes by the microorganism subjected to a low temperature stress $\left(5^{\circ} \mathrm{C}\right)$ in comparison with control $\left(15^{\circ} \mathrm{C}\right)$. The genes relating to lipid metabolism, especially the synthesis of LCPUFA, were focused on.

\section{Results and Discussion}

\subsection{Sequence Analysis and Transcriptome Annotation}

The total output of HiSeq 4000 for all the samples was approximately 174 million paired-end reads, with an average of 29 million reads for each sample. The quality filtering step retained over $99.5 \%$ of the reads (173.7 million), confirming an excellent sequencing performance and assuring a minimum of $3 \mathrm{Gbps}$ of data (20 million reads) per sample (Table S1, Supplemental Material). The final transcriptome resulted in 118,595 transcript sequences that were contained in 69,220 genes. The quality metrics indicated that $50 \%$ of the assembled bases were contained in transcripts longer than $2518 \mathrm{bp}$ (N50), with an average transcript length of $1243 \mathrm{bp}$, and a total size of $147 \mathrm{Mbp}$. The presence of several short transcripts in the assembly was evidenced by a median length metric of 554 bp (half of the assembled transcripts had a length of $554 \mathrm{bp}$ or less) and this may have affected the average length of the transcriptome (Table 1). The results from BUSCO (Benchmarking Universal Single-Copy Orthologs [17]) indicated a high degree of transcriptome completeness, resulting in 235 of the 255 genes used for evaluation being found (Table 2). 
Table 1. Assembly metrics for the transcriptome of Oblongichytrium RT2316-13.

\begin{tabular}{cc}
\hline Metric & Value \\
\hline Total raw reads (bp) & $174,570,852$ \\
Total high quality reads (bp) & $173,659,258$ \\
High quality content (\%) & 99.47 \\
Number of transcripts & 118,595 \\
Number of genes & 69,220 \\
Total size (Mbp) & 147.5 \\
N50 (bp) & 2518 \\
Average length (bp) & 1243 \\
Median length (bp) & 554 \\
\hline
\end{tabular}

Table 2. Summary of BUSCO ${ }^{\S}$ de novo transcriptome assembly of Oblongichytrim RT2316-13.

\begin{tabular}{ccc}
\hline Library $\$$ & Quantity & Percent of Total (\%) \\
\hline Complete BUSCOs & 223 & 87.4 \\
Single-copy BUSCOs & 125 & 49.0 \\
Duplicated BUSCOs & 98 & 38.4 \\
Fragmented BUSCOs & 12 & 4.7 \\
Missing BUSCOs & 20 & 7.9 \\
Total BUSCO genes & 255 & 100 \\
\hline
\end{tabular}

§ BUSCO (Benchmarking Universal Single-Copy Orthologs [17]).

Alignment of high quality reads to the assembled transcriptome of RT2316-13 showed a high mapping rate: an average of $91.63 \%$ for the properly aligned paired reads, and an overall mapping rate $>99 \%$ for every sample. The overall alignment included reads that contained one mismatch in their sequences, and reads that were aligned separately (Table S2, Supplemental Material).

\subsection{Differentially Expressed Genes}

Using a false discovery rate (FDR) of 0.05 and a fold change (FC) of 4 as thresholds, 2944 genes were found to be differentially expressed (DE). Of these DE genes, 2062 were induced by the low temperature (LT) whereas 882 genes were repressed (Excel file Paredes_et_al.xlsx, Supplemental Material). The homology searches in the public database SwissProt indicated that relatively few of the DE genes $(25.1 \%$ ) had significant matches, probably because of a comparative rarity of microbes of the genus Oblongichytrium. Of the annotated genes (1999 transcripts), 533 were induced and 206 were repressed (Excel file Paredes_et_al.xlsx, Supplemental Material). Most of the DE genes belonged to the GO-slim (cut-down versions of the Gene Ontologies) cellular component category (Figure 1a). The DE proteins were classified into 16 classes (Figure 1b). Among the induced proteins, the class "Nucleic acid binding" (PC00031, $p=0.0187$ ) was depleted, while "Cytoskeletal protein" (PC00085, $p=0.0006$ ) was overrepresented. In the repressed protein group the overrepresented classes were the "Translation protein" (PC00263, $p<0.0001$ ) and its subclass "Ribosomal protein" (PC00202, $p<0.0001$ ). The subclass "Oxidoreduction", whose parent was "Metabolite interconversion enzyme", was also overrepresented (PC00176, $p=0.0022$ ). Genes relating to carbohydrate, amino acids, energy, and lipid metabolism represented $64.4 \%$ of the annotated genes (Figure 1c). In the lipid metabolism group, the genes relating to fatty acid degradation, and metabolism of glycerophospholipid and glycerolipid, accounted for $44.5 \%$ of the annotated genes whereas $6.2 \%$ of the annotated genes related to fatty acid elongation (Figure 1d). Annotated genes related to carbohydrate metabolism (21\%, Figure 1e) were the most abundant and within this group more than $52 \%$ corresponded to genes coding for enzymes related to glycolysis, inositol phosphate, citrate cycle, and pyruvate metabolisms. 
a

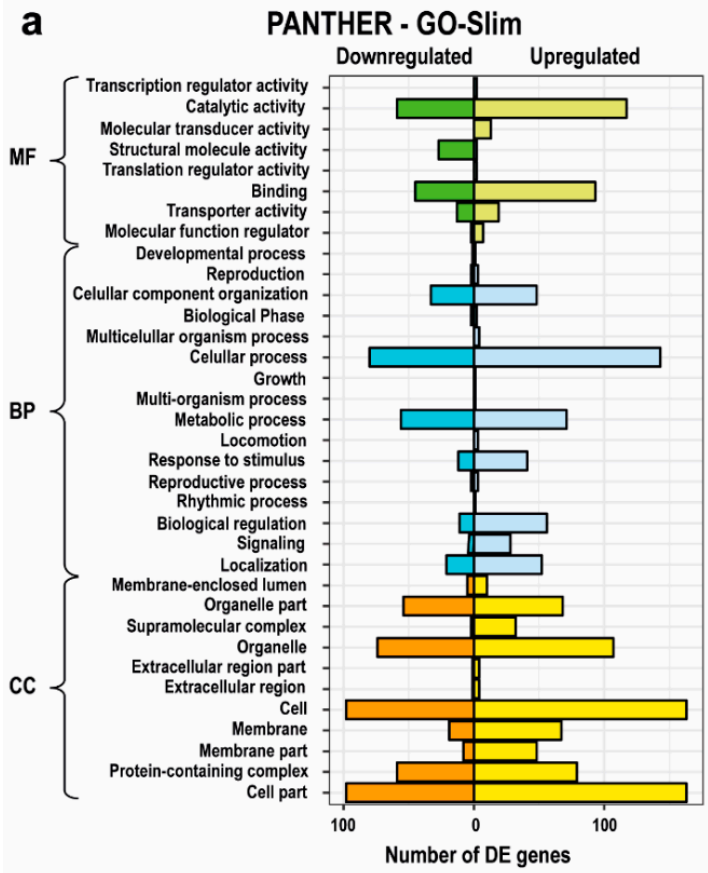

d

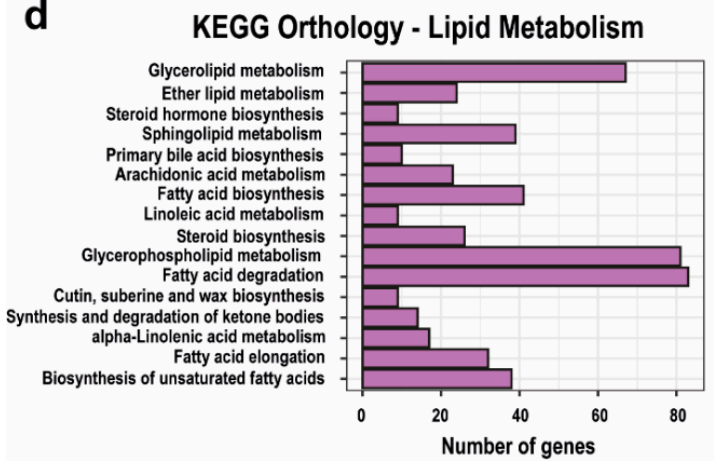

b
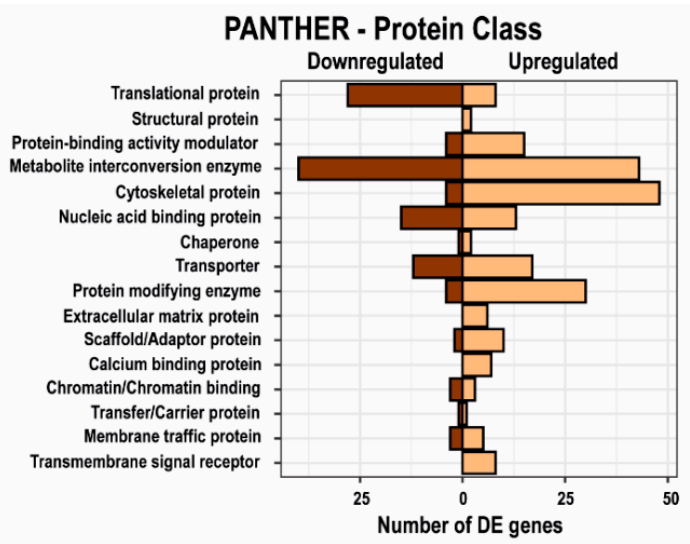

C

KEGG Orthology - Metabolism

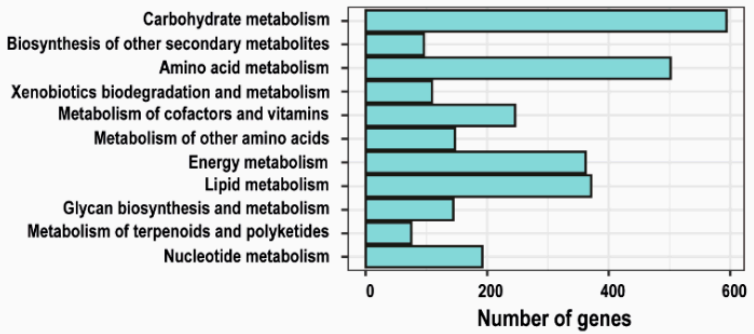

e

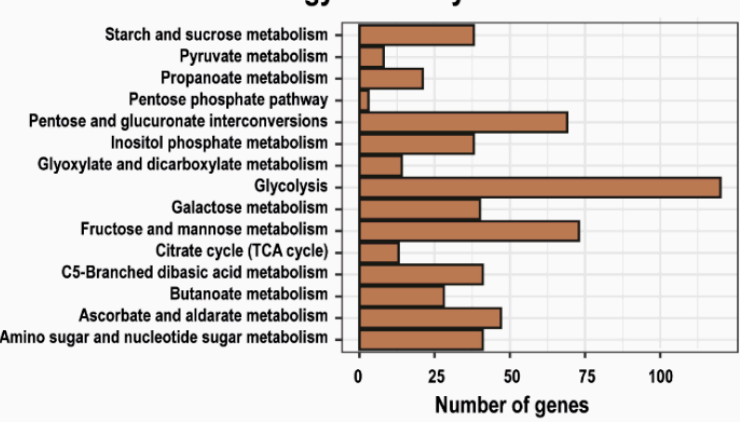

Figure 1. Number of differentially expressed (DE) genes in: (a) different GO-slim (cut-down versions of the Gene Ontologies) categories (molecular function, MF; biological process, BP; and cellular component, CC); and (b) different PANTHER protein classes. Distribution of the annotated genes in: (c) the different metabolisms; (d) lipid metabolism; and (e) carbohydrate metabolism, in the de novo assembled transcriptome of Oblongichytrium RT2316-13.

\subsubsection{Cytoskeletal Proteins}

Forty-eight genes coding for the cytoskeleton-related proteins were DE. The cytoskeleton of eukaryotes is a dynamic network of interlinking protein filaments that confer shape and mechanical resistance to cells, and allows them to move. Cytoskeleton is also involved in many cell signaling pathways and intracellular transport. The cytoskeleton is composed of microfilaments (linear polymers of G-actin proteins), intermediate filaments, and microtubules (MT, polymers of tubulin). In RT2316-13, the genes coding for tubulin $\alpha$-chain $(2$ genes $)(F C=10.88 ; 16.46)$, tubulin tyrosine ligase $(\mathrm{FC}=11.73)$, tubulin polymerization-promoting protein family member $(\mathrm{FC}=17.02)$, and actin ( 2 genes) $(\mathrm{FC}=8.47$; 27.03) were upregulated.

Cilia are evolutionarily conserved, MT-based organelles that play important roles in cell movement, environment sensing, and signal transduction. Motile biflagellate zoospores are characteristic of most thraustochytrids [18], including RT2316-13 (microscopic observation). Eighteen genes coding for cilia and flagella-associated proteins were upregulated (FC in the range of 5.34 to 18.25). The assembly, maintenance, and functions of cilia depend on the intraflagellar transport (IFT), the bidirectional 
trafficking of vesicles and proteins along the ciliary axoneme [19]. IFT is mediated by motor protein complexes, the cytoplasmic dyneins, and kinesins. In RT2313-13, the expression of genes coding for kinesin-related proteins (19 genes) (FC in the range of 4.41 to 15.84), and dynein-related proteins (24 genes) (FC in the range of 4.49 to 19.96) were upregulated.

The contribution of cytoskeleton to the cell's structural integrity, organization, and movement explained the significant number of the DE genes in RT2316-13 in response to a change in growth temperature. Most of the annotated genes were upregulated which suggested that the cells either needed to adapt or reshape their structure, or elevate the level of certain intracellular enzymes, to enable movement at the low temperature.

\subsubsection{Inositol Phosphate Metabolism}

Phosphatidylinositol (PI) is a family of lipids that are plentiful in cell membranes [20]. In one of its roles, PI modulates protein function, depending on the phosphorylation state of inositol. Deletion of INP51 (a gene coding a protein with 5-phosphatase activity against phosphatidylinositol 4,5-bisphosphate, PI(4,5)P2) in Saccharomyces cerevisiae resulted in accumulation of PI(4,5)P2 that correlated to cold-tolerance through an unknown mechanism [21]. In RT2316-13, the genes encoding phosphatidylinositol 4-phosphate 5-kinase ( $\mathrm{FC}=4.79 ; 7.92 ; 18.75)$ and phosphatidylinositol 4-kinase ( $\mathrm{FC}=8.19$ ), and the enzymes involved in PI $(4,5) \mathrm{P} 2$ synthesis, were upregulated, suggesting a role in cold-tolerance as previously observed in S. cerevisiae.

$\mathrm{PI}(4,5) \mathrm{P} 2$ is hydrolyzed by phospholipase C (PLC), an enzyme known to activate at low temperatures [22,23], to produce the secondary messengers diacylglycerol (DAG) and inositol-1,4,5-trisphosphate. In RT2316-13 a gene coding for a PLC (FC = 13.77) was upregulated. Once formed, DAG activated protein kinase C (PKC), an enzyme that has a central role in the cell wall integrity signaling pathway that responds to cell surface stress in S. cerevisiae [24]. In RT2316-13 a gene coding for a $\mathrm{Ca}^{2+}$-independent, phospholipid- and DAG-dependent serine/threonine-protein kinase (novel $\mathrm{PKC}$ ) was upregulated $(\mathrm{FC}=16.67)$. Upregulation of the genes relating to the synthesis of PI(4,5)P2, PLC and PKC suggested LT as a stress factor for RT2316-13.

\subsubsection{Glycolysis, Pentose Phosphate Pathway, and Tricarboxylic Acid Cycle}

Four genes coding for enzymes in the glycolysis pathway were upregulated by LT in RT2316-13. These genes related to phosphoglycerate kinase $(\mathrm{FC}=14.09)$, enolase $(\mathrm{FC}=8.81)$, phosphoglycerate mutase $(\mathrm{FC}=5.70)$, and phosphoenolpyruvate synthase $(\mathrm{FC}=6.24)$. Upregulation of the latter gene might enable cells to use three-carbon substrates (e.g., lactate, pyruvate, and alanine) as carbon sources [25]. The LT treatment upregulated a gene coding for a probable 6-phosphofructo-2-kinase (FC $=7.12$ ) in RT2316-13. This enzyme catalyzes the production of fructose-2,6-bisphosphate which allosterically activates 6-phosphofructo-1-kinase, a rate-limiting enzyme and an essential control point in the glycolytic pathway. Genes coding for 2,3-bisphosphoglycerate-dependent phosphoglycerate mutase $(\mathrm{FC}=-9.44)$, fructose-bisphosphate aldolase ( $\mathrm{FC}=-17.70)$, a NADP-dependent alcohol dehydrogenase $(\mathrm{FC}=-17.32)$, and acetyl-coenzyme A synthetase ( $\mathrm{FC}=-16.51$ ), were downregulated.

Four genes coding for enzymes in the pentose phosphate pathway were DE in RT2316-13. Among these, a gene coding for NADPH-producing 6-phosphogluconate dehydrogenase, a source of NADPH for fatty acid biosynthesis, was downregulated $(\mathrm{FC}=-17.01)$. On the other hand, genes coding for a ribose-phosphate pyrophosphokinase $4(\mathrm{FC}=4.30)$, involved in nucleotide biosynthesis, and ribokinase $(\mathrm{FC}=11.30)$ which catalyzes the phosphorylation of ribose to ribose-5-phosphate, were upregulated. The upregulation of the ribokinase gene could be related to a possible degradation of ribosomes to release nutrients [26]. The LT treatment downregulated 33 genes coding for ribosomal proteins in RT2316-13. This was because a large number of ribosomes were needed only during rapid growth [27] and the slowed growth at low temperature promoted catabolic recycling of the older ribosomes to release amino acids and nucleotides. Nucleotides could be dephosphorylated, and hydrolyzed to ribose and nucleobases [28]. The action of ribokinase produces ribose-5-phosphate that can be converted to 
glycolytic intermediates (fructose-6-phosphate and glyceraldehyde-3-phosphate) in the nonoxidative pentose phosphate pathway.

The LT treatment downregulated four genes coding for enzymes in the citric acid cycle (fumarate hydratase, $\mathrm{FC}=-19.48$; malate dehydrogenase, $\mathrm{FC}=-16.03$; succinateCoA ligase, $\mathrm{FC}=-14.91$; and aconitate hydratase, FC $=-14.67$ ), whereas a gene coding for isocitrate dehydrogenase [NADP+] $(F C=6.38)$ was upregulated. These results showed that the LT treatment depressed the activity of the TCA cycle, reducing energy supply and the production of citric acid. The decreased activity of the TCA cycle and NADPH-producing 6-phosphogluconate dehydrogenase explained the reduced lipid content (from $33.5 \%$ to $15.2 \% w / w$ ) in RT2316-13 biomass grown at $5{ }^{\circ} \mathrm{C}$ [14].

Other similar observations have been reported. An earlier study with Aurantiochytrium found a reduced supply of biochemical energy from glycolysis and TCA cycle, as a consequence of cold stress $\left(15^{\circ} \mathrm{C}\right.$ compared with $\left.25^{\circ} \mathrm{C}\right)$ [29]. The authors further observed that in the logarithmic growth phase, the expression of genes coding for a ribose 5-phosphate isomerase and ribose-phosphate pyrophosphokinase, the enzymes required for nucleotide synthesis, was upregulated.

\subsubsection{Respiratory Chain and Oxidative Phosphorylation}

Several genes of the respiratory chain were downregulated by the LT treatment. These were the genes coding for subunits of NADH dehydrogenase (ubiquinone) ( 3 genes) ( $\mathrm{FC}=-23.18 ;-17.32$; -15.98) (complex I), ATP synthase subunits (4 genes) (FC in the range of -17.36 to -15.37 ) (complex V), cytochrome coxidase subunit (4 genes) (FC in the range of -26.45 to -15.40) (complex IV), and ADP/ATP carrier protein (catalyzes the exchange of ADP and ATP across the mitochondrial inner membrane) $(F C=-19.26)$. The reduced activity of the respiratory chain and TCA cycle explained the reduced specific growth rate of RT2316-13 at the low temperature [14].

\subsubsection{Lipid Metabolism}

Two main pathways are responsible of the synthesis of eicosapentaenoic acid (EPA) and docosahexaenoic acid (DHA). These are the polyketide synthase (PKS) system found in marine bacteria (Shewanella sp., [30]; Vibrio sp., [31]; Photobacterium profundum, [32]) and in some thraustochytrids (Schizochytrium sp., [33], Thraustochytrium sp. SZU445 [34]), and the elongase-desaturase system (w3and $\omega 6$-pathways) (shown in Figure 2). Precursors of $\omega 6$ - and $\omega 3$-pathways (i.e. linoleic acid (LA, $\mathrm{C} 18: 2^{\Delta 9,12}$ ) and $\alpha$-linolenic (ALA, C18: $3^{\Delta 9,12,15}$ ), respectively), are synthetized from palmitic acid (PA, C16:0), the product of fatty acid synthase (FASN). Genes coding for FASN, $\Delta 9$ - and $\Delta 12$-desaturases were found in the transcriptome of RT2316-13 (Table 3); however, the expression of a gene coding for $\Delta 15$-desaturase ( $\omega 3$-desaturase), involved in ALA synthesis from LA, did not occur in RT2316-13 under the specified conditions. The trace ALA content $(<1 \% w / w$ of total fatty acids) in RT2316-13 (Figure 2) could be explained by a bifunctional $\Delta 12$-desaturase as has been shown to occur in some fungi [35]. 


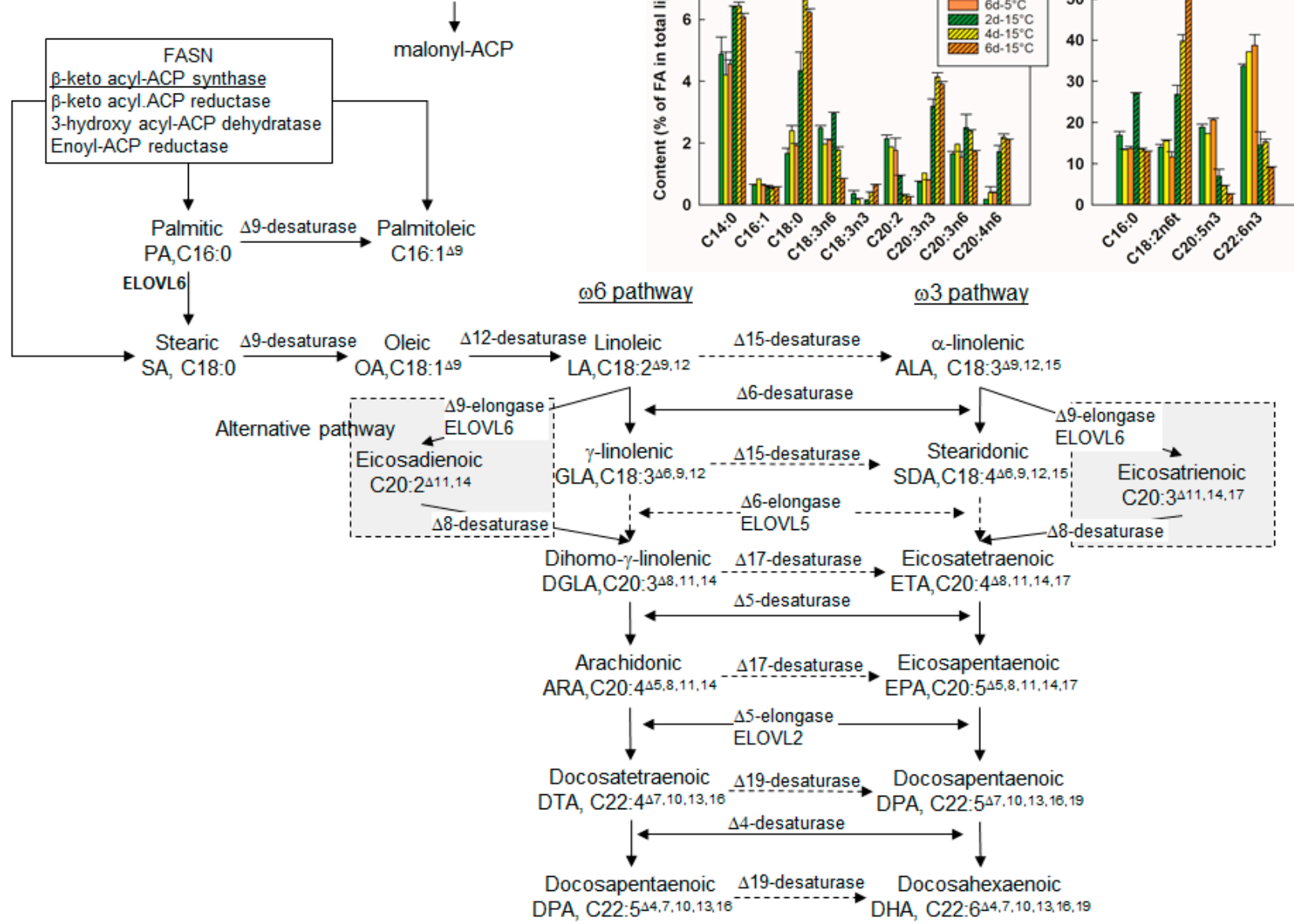

Figure 2. $\omega 3$ - and $\omega 6$-pathways of biosynthesis of long chain polyunsaturated fatty acids and the alternative pathway ( $\Delta 6$-elongase (ELOV6) $+\Delta 8$-desaturase) Reactions catalyzed by enzymes of the genes expressed in RT2316-13 are shown as continuous solid lines with arrowheads. Dashed lines with arrowheads denote reactions that would be catalyzed by enzymes/genes not expressed by RT2316-13 under the growth conditions. Inset graphs show the composition of the fatty acids in the total lipids of RT2316-13 at different temperatures $\left(5^{\circ} \mathrm{C}\right.$ and $\left.15^{\circ} \mathrm{C}\right)$ and periods of incubation $(2,4$ and 6 days) [11]. See reference [11] for further details. 
Table 3. Annotated genes in the transcriptome of Oblongichytrium RT2316-13 involved in the biosynthesis, elongation and desaturation of fatty acids.

\begin{tabular}{|c|c|c|}
\hline Enzyme (Reaction) & EC Number & Swiss Prot ID \\
\hline \multicolumn{3}{|l|}{ Fatty acid biosynthesis } \\
\hline Acetyl-CoA carboxylase & 6.4.1.2 & ACACB_HUMAN; ACAC_YEAST; ACAC_SCHPO; ACACA_MOUSE \\
\hline Fatty acid synthase, FASN & 2.3.1.85 & FAS_HUMAN \\
\hline Fatty acid synthase subunit $\beta$ & 2.3.1.86 & FAS1_CANAX \\
\hline Fatty acid synthase subunit $\alpha$ & 2.3.1.86 & FAS2_PENPA \\
\hline Fatty acid synthase $\beta$ subunit aflB & & ATNM_EMENI \\
\hline Malonyl CoA-acyl carrier protein transacylase & 2.3.1.39 & FABD_BACSU \\
\hline 3-Oxoacyl-[acyl-carrier-protein] reductase FabG & 1.1.1.100 & FABG_THEMA; FABG_RICPR; FABG_VIBCH \\
\hline 3-Oxoacyl-[acyl-carrier-protein] synthase * & 2.3.1.179 & KASM_ARATH \\
\hline Hydroxyacyl-thioester dehydratase type 2 & 4.2.1. & HTD2_HUMAN \\
\hline Fatty acyl-CoA synthetase A & 6.2.1.3 & FCSA_DICDI \\
\hline Very long-chain acyl-CoA synthetase & 6.2.1.3 & S27A2_MOUSE; S27A2_HUMAN \\
\hline Enoyl-CoA hydratase ACTT6 $\$$ & 5.3.3.14 & ACTT6_ALTAL \\
\hline \multicolumn{3}{|l|}{ Fatty acid elongation } \\
\hline 3-Ketoacyl-CoA thiolase & 2.3 .1 .16 & THIKB_RAT \\
\hline Trifunctional enzyme subunit $\beta$ & 2.3.1.16 & ECHB_MACFA; ECHB_BOVIN \\
\hline Trifunctional enzyme subunit $\alpha$ & 2.3.1.16 & ECHA_HUMAN; ECHA_PIG \\
\hline 3-Hydroxyacyl-CoA dehydrogenase & 1.1.1.35 & HCD2_DROME; HCDH2_CAEEL; HCDH1_CAEEL \\
\hline Enoyl-CoA hydratase & 4.2.1.17 & ECHM_BOVIN; ECHM_DICDI; ECHM_RAT \\
\hline Enoyl-[acyl-carrier-protein] reductase 1 & 1.3.1.; 1.3.1.38 & ETR1_DEBHA; MECR_DICDI \\
\hline Lysosomal thioesterase PPT2-A & 3.1.2.22 & PPT2A_XENLA \\
\hline Palmitoyl-protein thioesterase 1 & 3.1.2.22 & PPT1_MACFA \\
\hline Elongation of very long chain fatty acids protein 2 (ELOVL2) & 2.3.1.199 & ELOV2_HUMAN \\
\hline Elongation of very long chain fatty acids protein 4 (ELOVL4) $)^{£}$ & 2.3.1.199 & ELOV4_HUMAN̄; ELOV4_MACMU \\
\hline Elongation of very long chain fatty acids protein 6 (ELOVL6) $\ddagger$ & 2.3.1.199 & ELO6_CAEEL; ELOV6_DANRE; ELOV6_MOUSE \\
\hline Putative elongation of fatty acids protein & 2.3.1.199 & Y2012_DICDI \\
\hline Very-long-chain 3-oxoacyl-CoA reductase & 1.1.1.330 & MKAR_LACBS; KCR1_ARATH \\
\hline Very-long-chain (3R)-3-hydroxyacyl-CoA dehydratase & 4.2.1.134 & HACD_CAEEL \\
\hline Very-long-chain enoyl-CoA reductase & 1.3.1.93 & TECR_ARATḦ; TECR_DICDI \\
\hline Cytosolic acyl coenzyme A thioester hydrolase & 3.1.2.2 & BACH_HUMAN; BACH_RAT \\
\hline
\end{tabular}


Table 3. Cont

\begin{tabular}{ccc}
\hline Enzyme (Reaction) & EC Number & Swiss Prot ID \\
\hline Biosynthesis of unsaturated fatty acids & & ACOD2_MOUSE \\
\hline Acyl-CoA desaturase $(\Delta$ 9 desaturase) & 1.14 .19 .1 & SCD1_TACFU \\
Acyl-CoA desaturase 1 & 1.14 .19 .1 & 1.14 .19 .3 \\
Acyl-CoA 6-desaturase & 1.14 .19 .6 & LLCD_SYNY3; FADS2_PONAB \\
Delta(12) fatty acid desaturase FAD2 & 1.14 .19 .31 & FAD2_CALOF \\
Acyl-lipid (7-3)-desaturase ( $\Delta 4$ desaturase) & $1.14 .19 .17 ; 1.14 .18 .5$ & D4FAD_EUGGR;D4FAD_THRSP \\
Sphingolipid delta(4)-desaturase & 1.14 .19 .3 & DEGS_KOMPG; DEGS_CANAL \\
Delta(8)-fatty-acid desaturase & 3.1 .2$. & SLD1_EUGGR * SLD2_ARATH \\
Acyl-CoA thioesterase 2 & TESB_ECOLI \\
\hline
\end{tabular}

* Substrate specificity of this enzyme is similar to that of EC 2.3.1.41, but it differs in that palmitoleoyl-ACP is not a good substrate for it. This enzyme controls the temperature-dependent regulation of fatty-acid composition in Escherichia coli [36]. \$ The cis-3-enoyl product is required to form unsaturated fatty acids such as palmitoleic acid and cis-vaccenic acid, in dissociated (or type II) fatty-acid biosynthesis. ${ }^{¥}$ Acts specifically on polyunsaturated acyl-CoA with a higher activity toward C20:4 ${ }^{45,8,11,14}$ and EPA-CoAs, among others [37]. Other substrates include DTA-CoA, EPA-CoA, DPA-CoA. ELOVL2 elongates. ${ }^{£}$ ELOLV4 substrates: DTA-CoA, C26:4n6-CoA, C28:4n6-CoA, C30:4n6-CoA, C32:4n6-CoA, C34:4n6-CoA, C34:6n6-CoA, C24:0-CoA, C26:0-CoA, C28:0-CoA, C30:0-CoA, DHA-CoA, C24:5n3-CoA, C24:6n3-CoA, C26:5n3-CoA, C26:6n3-CoA, C28:5n3-CoA, C28:6n3-CoA, C30:5n3-CoA, C30:6n3-CoA, C32:5n3-CoA,

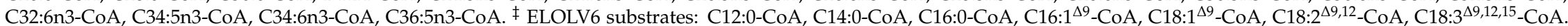

${ }^{*}$ Transcript TRINITY_DN4828_c0_g2_i1 was translated to protein, and queried by homology against non-redundant protein database in National Center for Biotechnology Information (NCBI) using the protein BLAST (Basic Local Alignment Search Tool) algorithm (https://blast.ncbi.nlm.nih.gov). The results showed a high identity (59.62\%) and similarity (74.11\%) with a $\Delta 5$-desaturase of Oblongichytrium sp. SEK 347 (accession BAG71007.1) 
Genes coding for $\Delta 4-, \Delta 6$ - and $\Delta 8$-desaturases, and elongases were expressed in RT2316-13 (Table 3). Although fatty acid elongation occurs through sequential action of four enzymes (condensation, reduction, dehydration, and reduction) adding two carbon units to the growing acyl chain, the rate and substrate specificity are governed by the first reaction, catalyzed by a fatty acid elongase. The elongases expressed by RT2316-13 were similar to the human elongases (ELOVL2, ELOVL4, and ELOVL6) (Table3). The $\omega 3$ - and $\omega 6$-pathways require $\Delta 17$ - and $\Delta 19$-desaturases ( $\omega 3$-desaturases) for the synthesis of DHA from the $\omega 6$ fatty acid substrates, but these enzymes were missing in RT2316-13. A key enzyme required for the synthesis of arachidonic acid (ARA, C20:4 $4^{\Delta 5,8,11,14}$ ), EPA and DHA through the $\omega 3$ and w6-pathways, is $\Delta 5$-desaturase. The transcript TRINITY_DN4828_c0_g2_i1 identified as a $\Delta 8$-fatty acid desaturase (SLD1_EUGGR, Table 3) was translated to protein, and queried by homology against non-redundant protein database in National Center for Biotechnology Information (NCBI) using the protein BLAST (Basic Local Alignment Search Tool) algorithm (https://blast.ncbi.nlm.nih.gov). The results showed a high identity $(59.62 \%)$ and similarity $(74.11 \%)$ with a $\Delta 5$-desaturase from Oblongichytrium sp. SEK 347 (accession BAG71007.1).

A homology search for genes in the PKS system involved in the EPA and/or DHA biosynthesis ( $p f a A, p f a B, p f a C$, and $p f a D$ ) in the transcriptome of RT2316-13 was performed. The one significant hit after being extracted and queried by homology on GenBank and UniProt databases matched to a polyketide synthase (PKS) gene related to antibiotic biosynthesis. Thus, under the growth conditions, LCPUFA synthesis in RT2316-13 was not due to the PKS system, unlike in the related thraustochytrids Schizochytrium sp. [33] and Thraustochytrium sp. SZU445 [34].

Based on the enzymes identified in RT2316-13, the synthesis of EPA, DHA, and ARA starts from palmitic acid (Figure 2). The sequential action of ELOVL 6 (a fatty acid elongase), $\Delta 9$-desaturase and $\Delta 12$-desaturase produces stearic acid, oleic acid, and linoleic acid, whereas the action of a bifunctional $\Delta 12 / \Delta 15$-desaturase on linoleic acid produces $\alpha$-linolenic acid. The latter is transformed into eicosatrienoic acid, eicosatetraenoic acid, and EPA by the sequential action of $\Delta 9$-elongase, $\Delta 8$-desaturase, and $\Delta 5$-desaturase. The action of $\Delta 5$-elongase and $\Delta 4$-desaturase on EPA and DPA, respectively, produces DHA. The action of $\Delta 9$-elongase, $\Delta 8$-desaturase, and $\Delta 5$-desaturase on linoleic acid, eicosadienoic acid, and dihomo- $\gamma$-linolenic produces ARA.

Twenty-five genes coding for enzymes related to glycerolipid metabolism and biosynthesis of fatty acids and steroids were DE in RT2316-13. Among the DE genes coding for enzymes involved in the synthesis of PUFA were those coding for: a very-long-chain 3-oxoacyl-CoA reductase $(\mathrm{FC}=8.32)$, a long-chain fatty acid CoA ligase (2 genes) ( $\mathrm{FC}=4.35 ; 6.08)$, ELOVL6 ( $\mathrm{FC}=6.41)$, ELOV2 (FC = 5.29), and a $\Delta 8$-fatty-acid desaturase $(F C=5.10)$. The latter gene was found to be highly similar to a $\Delta 5$-desaturase, a key enzyme in the synthesis of EPA and DHA. Upregulation of this gene together with upregulation of the gene coding for ELOV2 (a $\Delta 5$-elongase) explained the enrichment of the total lipid in EPA and DHA at LT (Figure 2).

Two genes coding for diacylglycerol O-acyltransferase 2, the enzyme required for the synthesis of triacylglycerols (TAG), were DE (FC $=14.62 ;-6.88)$, whereas two genes coding for monoacylglycerol lipase (MAGL) were upregulated ( $\mathrm{FC}=5.63 ; 12.24)$. MAGL catalyzes the last step in the hydrolysis of storage TAG and has been postulated to have a role in the detoxification of deleterious membrane lipids [38] which could form through oxidation of membrane PUFA because of the increased solubility of oxygen in water at LT $[39,40]$.

In RT2316-13, a gene coding for cardiolipin synthase A was upregulated $(\mathrm{FC}=4.60)$. Cardiolipins are phospholipids of the inner mitochondrial membrane required for the optimal activity of oxidative phosphorylation complexes and the ATP/ADP carrier [41]. Interactions of sterols with other membrane components contribute not only to regulate membrane fluidity and permeability, but also to the activity of membrane-bound enzymes and transporters [42]. Disruption of the SYR1/ERG3 gene that encoded sterol C-5 desaturase, an enzyme involved in the ergosterol biosynthesis, inhibited the growth of S. cereviciae at LT possibly because sterol was required for proper functioning of the 
tryptophan transporter at LT [43]. A similar function could explain the upregulation (FC $=20.09)$ of a $\Delta 7$-sterol-C5(6)-desaturase, involved in the biosynthesis of sitosterol and campesterol in RT2316-13.

\subsection{Validation of Gene Expression Profiles by $q R T-P C R$}

The amplification of four selected genes that coded for acetyl-CoA carboxylase (AAC-5, AAC-15), malic enzyme (Me-5, Me-15), subunit A of PKS (Pfaa-5, Pfaa-15), and subunit C of LCPUFA PKS (Pfac-5, Pfac-15), in cDNA of the biomass grown at $5{ }^{\circ} \mathrm{C}$ and $15^{\circ} \mathrm{C}$ was evaluated. The genes for AAC and Me were selected because the first of these is involved in the synthesis of fatty acid precursors, and the second one is involved in the synthesis of NADPH required for the synthesis of PUFA. Genes coding for acetyl-CoA carboxylase and malic enzyme were expressed at both the low and control temperatures (Figure S1). The lack of expression of the genes coding for the two subunits of LCPUFA PKS (Figure S1, Supplemental Material) was consistent with the results of transcriptome analysis. Five other genes coding for acetyl-CoA carboxylase and four other genes coding for malic enzyme were annotated in the transcriptome of RT2316-13, but these were not differentially expressed.

\section{Materials and Methods}

\subsection{Microorganism and Culture Conditions for RNA Extraction}

The thraustochytrid Oblongichytrium sp. RT2316-13 used in this work had been isolated from seawater samples collected near the Antarctic Base Professor Julio Escudero ( $62^{\circ} 12^{\prime} 57^{\prime \prime}$ S, 58 $57^{\prime} 35^{\prime \prime}$ E). Details of isolation and identification were previously published [15]. Briefly, the samples were supplemented with sterile pine pollen $(\sim 15 \mathrm{mg}$ per $200 \mathrm{~mL})$ and kept at $5{ }^{\circ} \mathrm{C}$. After 15 days, pollen grains were collected by filtration $(0.45 \mu \mathrm{m}$ pore size) and dispersed on a solid medium supplemented with streptomycin sulfate and penicillin G (0.3 g/L of each) (Sigma, St. Louis, MO, USA) to prevent proliferation of bacteria. Pure isolates were obtained through repeated subculture of individual colonies on solid medium. Cell DNA was isolated and the sequence of the $18 \mathrm{~S}$ rRNA gene was amplified using the method described previously [15]. Results of the Sanger sequencing were assembled using the Geneious 4.8.4 program (Biomatters Ltd., Auckland, New Zealand) and compared with sequences in EMBL/DDBJ/PDB/GenBank databases by BLASTN 2.2.21. Pure stock cultures were kept frozen at $-80{ }^{\circ} \mathrm{C}$ in $50 \%$ glycerol $(v / v)$.

Cells were grown aseptically. The culture medium comprised of glucose $\left(20 \mathrm{gL}^{-1}\right.$; Merck, Darmstadt, Germany), yeast extract (10 gL ${ }^{-1}$; BBL ${ }^{\mathrm{TM}}$, Becton, Dickinson and Co., Sparks, MD, USA), and monosodium glutamate $\left(0.6 \mathrm{gL}^{-1}\right.$; Merck) in a 1:1 by volume mixture of artificial sea water (ASW) [44] and distilled water. Trace elements and vitamins were added to the medium as previously specified [45]. Prior work had shown this medium to support growth with a highest attainable total lipid content in the biomass ( $35 \%$ of dry weight) at $15^{\circ} \mathrm{C}$ [14].

Inoculum was prepared by transferring $1 \mathrm{~mL}$ of the thawed stock to $100 \mathrm{~mL}$ of the earlier specified liquid medium in a $250 \mathrm{~mL}$ Erlenmeyer flask. The flask was incubated $\left(15^{\circ} \mathrm{C}\right)$ on an orbital shaker at $150 \mathrm{rpm}$ for 7 days. This culture $(5 \mathrm{~mL})$ was used to inoculate $100 \mathrm{~mL}$ of the same fresh medium and incubated $\left(15^{\circ} \mathrm{C}, 150 \mathrm{rpm}\right)$ for 2 days. Afterwards, $50 \mathrm{~mL}$ aliquots of the grown culture were transferred to two flasks, each containing $50 \mathrm{~mL}$ of the same fresh medium. One of the flasks was incubated at $15^{\circ} \mathrm{C}$ (control culture) and the other was incubated at $5{ }^{\circ} \mathrm{C}$ (low temperature (LT) culture) in an orbital shaker at $150 \mathrm{rpm}$. Both flasks were harvested after 3 days.

Biomass was harvested by centrifugation $\left(7000 \times g, 4{ }^{\circ} \mathrm{C}, 10 \mathrm{~min}\right)$. The recovered cell pellet was suspended in $12 \mathrm{~mL}$ of a 19:1 (by vol) mixture of aqueous phenol $(0.8 \mathrm{M})$ and absolute ethanol, and stored at $-20^{\circ} \mathrm{C}$ until the extraction of the nucleic acids. Three replicate experiments were used to produce the control and LT biomass samples. 


\subsection{RNA Extraction}

Cells from the previous step were lysed in a high-pressure homogenizer (Stansted Fluid Power Ltd., London, England) (one pass, 20 bar) under sterile conditions. Cell debris was removed from the lysate by centrifugation $\left(10,000 \times g, 4{ }^{\circ} \mathrm{C}, 15 \mathrm{~min}\right)$. Total RNA was extracted using TRIzol reagent (Thermo Fisher Scientific Inc., Waltham, MA, USA) according to the manufacturer's instructions. The collected RNA was purified using RNA purification columns (Thermo Fisher Scientific Inc., Waltham, MA, USA) following the manufacturer's instructions.

Sample degradation and integrity were monitored by agarose gel electrophoresis $(1 \% w / v, 100 \mathrm{mV}$, $30 \mathrm{~min}$ ). The purity and recovery yield of RNA were calculated using the absorbance values measured at 260 and $280 \mathrm{~nm}$ using a UV spectrophotometer (BioTek Synergy HT Microplate Reader; BioTek Instruments, Inc., Winooski, VT, USA).

\subsection{Conversion of RNA to cDNA}

The extracted RNA was treated with DNase to eliminate any contamination. One $\mu \mathrm{L}$ of random primers, mainly random hexanucleotides ( $500 \mu \mathrm{g} \mathrm{mL}^{-1}$; Promega Corporation, Madison, WI, USA) were added. A conversion mix (M-MLV RT 5x, M-MLV RT transcriptase, RNasin and dNTPs) was added. The first PCR program (MultiGene optiMAX; Labnet International Inc., Edison, NJ, USA) was: $5 \mathrm{~min}$ at $20^{\circ} \mathrm{C} ; 45 \mathrm{~min}$ at $37^{\circ} \mathrm{C} ; 5 \mathrm{~min}$ at $95^{\circ} \mathrm{C}$; and $4{ }^{\circ} \mathrm{C}$ until storage. A PCR using the primers for $18 \mathrm{~S}$ rRNA subunit (Table S3, Supplemental Material) was performed to verify the correct conversion. Conditions of the second PCR program were: $3 \mathrm{~min}$ at $95^{\circ} \mathrm{C} ; 30$ cycles of $1 \mathrm{~min}$ each at $94{ }^{\circ} \mathrm{C}$ followed by $1 \mathrm{~min}$ at $61^{\circ} \mathrm{C}$, and $1 \mathrm{~min}$ at $72{ }^{\circ} \mathrm{C} ; 10 \mathrm{~min}$ at $72{ }^{\circ} \mathrm{C}$; and $4{ }^{\circ} \mathrm{C}$ until storage. The cDNA was stored for validation assays.

\subsection{Sequencing and Quality Control of RT2316-13 Transcriptome}

A total of six samples were sequenced (Genoma Mayor, Santiago, Chile) in an Illumina HiSeq 4000 (Illumina, Inc., San Diego, CA, USA) platform for 150 cycles in paired-end mode. The sequencing outputs, in FastQ file format, were processed using TrimGalore software to remove adaptors and reads that were likely to be sequencing errors. The reads, which on average had a Phred score of less than 30 ( 1 error in $1000 \mathrm{bp}$ ), were removed from downstream analysis.

The remaining high quality reads, selected during the quality control step, were used to construct a de novo assembly of RT2316-13 transcriptome using the Trinity v2.9.1 software [46]. The software used de Bruijn graphs to reconstruct transcripts and their variants using an extensive K-mer search strategy. The assembly integrity was assessed using a set of conserved orthologous gene sequences contained in the Eukaryota OrthoDB v10 database (www.orthodb.org), which were queried by similarity to the assembled transcripts, as in the BUSCO approach (Benchmarking Universal Single-Copy Orthologs) described by Simão et al. [17].

\subsection{Differential Gene Expression Analysis}

The relative abundance was calculated using RSEM v1.2.26 [47]. The resulting abundance for each sample was merged in a matrix, and analyzed with the Bioconductor package edgeR in the $\mathrm{R}$ statistical environment [48]. The significance of changes in gene expression was judged using a False Discovery Rate value (FDR) of less than 0.05 and a minimum fold change (FC) of 4 as thresholds.

\subsection{Transcriptome Annotation and Enrichment Analysis}

All resulting genes were aligned into the UniProt/SwissProtKB database using BLAST + with an e-value of $1 \mathrm{e}^{-10}$ as the threshold. Functional annotation and ontology assignments were performed using the PANTHER classification system [49] with gene lists obtained from blast results (top hit) aligned with the reference proteome database (version 2018_4) from the European Molecular Biology 
Laboratory (EMBL). To search for enrichment or depletion of an ontology term among differentially expressed genes, they were analyzed using GOSeq [50].

\subsection{Validation of the Transcriptome Results}

Conventional PCR was performed to confirm or discard the presence of genes associated with LCPUFA synthesis. Five pairs of primers were used: Subunits A and C of PKS, malic enzyme, acetyl-CoA carboxylase, and, as a housekeeping gene, the subunit $18 \mathrm{~S}$ rRNA. The sequences were obtained from Ma et al. [29] (Table S1, Supplemental Material). Green Master Mix (Promega, Madison, WI, USA) was used for PCR in accordance with the manufacturer's instructions. The reaction was carried out in a final volume of $20 \mu \mathrm{L}$ using $4 \mu \mathrm{L}$ of cDNA synthesized as described above. Conditions of PCR program 2 (described above) were used. Results were verified by agarose gel $(1 \% w / v)$ electrophoresis.

\subsection{Data Availability}

The raw reads of the Sequence Read Archive (codes from SRR12600987 to SRR12600992) have been deposited at NCBI BioProject (accession number PRJNA642733).

\section{Conclusions}

This study contributes to knowledge of the cold water marine thraustochytrid Oblongichytrium RT2316-13. This microorganism grows abundantly at $5{ }^{\circ} \mathrm{C}$, a feature that is at least partly attributed to its ability to synthesize long chain omega-3 PUFA. Transcriptome analysis of Oblongichytrium RT2316-13 revealed a significant number of differentially expressed (DE) genes (2944) in response to a decrease in temperature. Most of the DE genes related to the composition of the cell membrane (fatty acids, sterols, phosphatidylinositol), the membrane-associated enzymes, and the membrane-linked cytoskeletal proteins and enzymes. This suggests that in this thraustochytrid, the cell membrane has a role in "sensing" temperature and is an active part of the temperature signal transduction system.

Thraustochytrids are mainly studied because of their capability to synthesize docosahexaenoic acid (DHA). However, strains such as RT2316-13 produce lipids rich in both EPA and DHA, fatty acids with known health effects. An understanding of temperature modulation of the genes related to the biosynthesis of these fatty acids is important for designing efficient production processes. The present work shows that in RT2316-13 the synthesis of EPA and DHA occurs through the action of elongases and desaturases and thus differs from synthesis in Aurantiochytrium species. The upregulation of genes coding for a $\Delta 5$-desaturase and $\Delta 5$-elongase involved in the synthesis of EPA and DHA explained the enrichment of total lipid with these two long chain polyunsaturated fatty acids at the low temperature.

Supplementary Materials: The following are available online at http://www.mdpi.com/1660-3397/18/11/563/s1, Figure S1: Amplification of cDNA segments. AAC, acetyl-CoA carboxylase; Me, malic enzyme; Pfaa, subunit A of PKS gene; Pfac, subunit C of PKS gene; 18s, $18 S$ subunit of RNA. xx-5 and xx-15 denote cDNA of cells grown at $5{ }^{\circ} \mathrm{C}$ and $15{ }^{\circ} \mathrm{C}$, respectively, Table S1: Sequencing results. The initial amount of reads (total reads) and the number of reads that passed quality control filters (HQ reads); Table S2: Total number of high quality (HQ) reads that were mapped to a sequence in RT2316-13 transcriptome, concordantly one or more times (properly aligned), and the total number of reads aligned (overall aligned), with their respective mapping rate (\%); Table S3: Primers used for identifying genes related to production of fatty acids, Excel file: Paredes_et_al.xlsx.

Author Contributions: C.S. conceived the research project leading to this publication. G.L. performed bioinformatics. P.P., A.L., L.F., and C.G.I. performed the experiments and prepared the initial draft of the manuscript. C.S., J.A.A., and Y.C. discussed the results and wrote the final manuscript. All authors have read and agreed to the published version of the manuscript.

Funding: This research was funded by Instituto Antártico Chileno (INACH), project Inach RT 23-16, and Conicyt, project Centre for Biotechnology and Bioengineering (CeBiB) FB-0001.

Acknowledgments: Dirección de Investigación, Universidad de La Frontera for GAP funding and undergraduate program.

Conflicts of Interest: The authors declare no conflict of interest. 


\section{References}

1. Kuratko, C.N.; Salem, N., Jr. Docosahexaenoic acid from algal oil. Eur. J. Lipid Sci. Technol. 2013, 115, 965-976. [CrossRef]

2. Marchan, L.F.; Chang, K.J.L.; Nichols, P.D.; Mitchell, W.J.; Polglase, J.L.; Gutierrez, T. Taxonomy, ecology and biotechnological applications of thraustochytrids: A Review. Biotechnol. Adv. 2018, 36, 26-46. [CrossRef] [PubMed]

3. Narayan, B.; Miyashita, K.; Hosakawa, M. Physiological effects of eicosapentaenoic acid (EPA) and docosahexaenoic acid (DHA)—A Review. Food Rev. Int. 2006, 22, 291-307. [CrossRef]

4. Allen, E.E.; Facciotti, D.; Bartlett, D.H. Monounsaturated but not polyunsaturated fatty acids are required for growth of the deep-sea bacterium Photobacterium profundum SS9 at high pressure and low temperature. Appl. Environ. Microbiol. 1999, 65, 1710-1720. [CrossRef] [PubMed]

5. Kato, C.; Nogi, Y. Correlation between phylogenetic structure and function: Examples from deep-sea Shewanella. FEMS Microbiol. Ecol. 2001, 35, 223-230. [CrossRef] [PubMed]

6. Kawamoto, J.; Kurihara, T.; Yamamoto, K.; Nagayasu, M.; Tani, Y.; Mihara, H.; Hosokawa, M.; Baba, T.; Sato, S.B.; Esaki, N. Eicosapentaenoic acid plays a beneficial role in membrane organization and cell division of a cold-adapted bacterium, Shewanella livingstonensis Ac10. J. Bacteriol. 2009, 191, 632-640. [CrossRef]

7. Stillwell, W.; Wassall, S.R. Docosahexaenoic acid: Membrane properties of a unique fatty acid. Chem. Phys. Lipids 2003, 126, 1-27. [CrossRef]

8. Cullen, J.; Phillips, M.C.; Shipley, G.G. The effects of temperature on the composition and physical properties of the lipids of Pseudomonas fluorescens. Biochem. J. 1971, 125, 733-742. [CrossRef] [PubMed]

9. Mansilla, M.C.; Cybulski, L.E.; Albanesi, D.; de Mendoza, D. Control of membrane lipid fluidity by molecular thermosensors. J. Bacteriol. 2004, 186, 6681-6688. [CrossRef] [PubMed]

10. Wada, H.; Murata, N. Membrane lipids in Cyanobacteria. In Lipids in Photosynthesis: Structure, Function and Genetics; Siegenthaler, P.-A., Murata, N., Eds.; Kluwer Academic Publishers: Dordrecht, The Netherland, 1998; pp. 65-81. [CrossRef]

11. Boelen, P.; van Dijk, R.; Damsté, J.S.S.; Rijpstra, W.I.C.; Buma, A.G.J. On the potential application of polar and temperate marine microalgae for EPA and DHA production. AMB Express 2013, 3, 26. [CrossRef] [PubMed]

12. Fan, K.W.; Jiang, Y.; Faan, Y.W.; Chen, F. Lipid characterization of mangrove thraustochytrid-Schizochytrium mangrovei. J. Agric. Food Chem. 2007, 55, 2906-2910. [CrossRef] [PubMed]

13. Okuyama, H.; Orikasa, Y.; Nishida, T. In Vivo conversion of triacylglycerol to docosahexaenoic acid-containing phospholipids in a thraustochytrid-like microorganism, strain 12B. Biotechnol. Lett. 2007, 29, 1977-1981. [CrossRef] [PubMed]

14. Shene, C.; Paredes, P.; Flores, L.; Leyton, A.; Asenjo, J.A.; Chisti, Y. Dynamic flux balance analysis of biomass and lipid production by Antarctic thraustochytrid Oblongichytrium sp. RT2316-13. Biotechnol. Bioeng. 2020, 117, 3006-3017. [CrossRef] [PubMed]

15. Shene, C.; Paredes, P.; Vergara, D.; Leyton, A.; Garcés, M.; Flores, L.; Rubilar, M.; Bustamante, M.; Armenta, R. Antarctic thraustochytrids: Producers of long chain omega-3 polyunsaturated fatty acids. Microbiologyopen 2019, 9, e950. [CrossRef] [PubMed]

16. Morita, R.Y. Psychrophilic bacteria. Bacteriol. Rev. 1975, 39, 144-167. [CrossRef] [PubMed]

17. Simão, F.A.; Waterhouse, R.M.; Ioannidis, P.; Kriventseva, E.V.; Zdobnov, E.M. BUSCO: Assessing genome assembly and annotation completeness with single-copy orthologs. Bioinformatics 2015, 31, 3210-3212. [CrossRef]

18. Moss, S.T. (Ed.) Biology and phylogeny of the Labyrinthulales and Thraustochytriales. In Biology of Marine Fungi; Cambridge University Press: Cambridge, UK, 1986; pp. 105-130.

19. Hao, L.; Scholey, J.M. Intraflagellar transport at a glance. J. Cell Sci. 2009, 122, 889-892. [CrossRef]

20. Martin, T.F.J. Phosphoinositide lipids as signaling molecules: Common themes for signal transduction, cytoskeletal regulation, and membrane trafficking. Annu. Rev. Cell Dev. Biol. 1998, 14, 231-264. [CrossRef]

21. Stolz, L.E.; Kuo, W.J.; Longchamps, J.; Sekhon, M.K.; York, J.D. INP51, a yeast inositol polyphosphate 5-phosphatase required for phosphatidylinositol 4,5-bisphosphate homeostasis and whose absence confers a cold-resistant phenotype. J. Biol. Chem. 1998, 273, 11852-11861. [CrossRef] 
22. Ruelland, E.; Cantrel, C.; Gawer, M.; Kader, J.C.; Zachowski, A. Activation of phospholipases C and D is an early response to a cold exposure in Arabidopsis suspension cells. Plant Physiol. 2002, 130, 999-1007. [CrossRef]

23. Schade, A.; Walliser, C.; Wist, M.; Haas, J.; Vatter, P.; Kraus, J.M.; Filingeri, D.; Havenith, G.; Kestler, H.A.; Milner, J.D.; et al. Cool-temperature-mediated activation of phospholipase C- $\gamma 2$ in the human hereditary disease PLAID. Cell. Signal. 2016, 28, 1237-1251. [CrossRef] [PubMed]

24. Levin, D.E. Cell wall integrity signaling in Saccharomyces cerevisiae. Microbiol. Mol. Biol. R. 2005, 69, $262-291$. [CrossRef] [PubMed]

25. Cooper, R.A.; Kornberg, H.L. Net formation of phosphoenolpyruvate from pyruvate by Escherichia coli. Biochim. Biophys. Acta 1965, 104, 618-620. [CrossRef]

26. Xu, Y.F.; Létisse, F.; Absalan, F.; Lu, W.; Kuznetsova, E.; Brown, G.; Caudy, A.A.; Yakunin, A.F.; Broach, J.R.; Rabinowitz, J.D. Nucleotide degradation and ribose salvage in yeast. Mol. Syst. Biol. 2013, 9, 665. [CrossRef]

27. Mager, W.H.; Planta, R.J. Coordinate expression of ribosomal protein genes in yeast as a function of cellular growth rate. Mol. Cell. Biochem. 1991, 104, 181-187. [CrossRef] [PubMed]

28. Tozzi, M.G.; Camici, M.; Mascia, L.; Sgarrella, F.; Ipata, P.L. Pentose phosphates in nucleoside interconversion and catabolism. FEBS J. 2006, 273, 1089-1101. [CrossRef]

29. Ma, Z.; Tan, Y.; Cui, G.; Feng, Y.; Cui, Q.; Song, X. Transcriptome and gene expression analysis of DHA producer Aurantiochytrium under low temperature conditions. Sci. Rep. 2015, 5, 14446. [CrossRef]

30. Yazawa, K. Production of eicosapentaenoic acid from marine bacteria. Lipids 1996, 31, S297-S300. [CrossRef]

31. Tanaka, M.; Ueno, A.; Kawasaki, K.; Yumoto, I.; Ohgiya, S.; Hoshino, T.; Ishizaki, K.; Okuyama, H.; Morita, N. Isolation of clustered genes that are notably homologous to the eicosapentaenoic acid biosynthesis gene cluster from the docosahexaenoic acid-producing bacterium Vibrio marinus strain MP-1. Biotechnol. Lett. 1999, 21, 939-945. [CrossRef]

32. Allen, E.E.; Bartlett, D.H. Structure and regulation of the omega-3 polyunsaturated fatty acid synthase genes from the deep-sea bacterium Photobacterium profundum strain SS9. Microbiology 2002, 148, 1903-1913. [CrossRef]

33. Metz, J.G.; Roessler, P.; Facciotti, D.; Levering, C.; Dittrich, F.; Lassner, M.; Valentine, R.; Lardizabal, K.; Domergue, F.; Yamada, A.; et al. Production of polyunsaturated fatty acids by polyketide synthases in both prokaryotes and eukaryotes. Science 2001, 293, 290-293. [CrossRef] [PubMed]

34. Zhu, X.; Li, S.; Liu, L.; Li, S.; Luo, Y.; Lv, C.; Wang, B.; Cheng, C.H.K.; Chen, H.; Yang, X. Genome sequencing and analysis of Thraustochytriidae sp. SZU445 provides novel insights into the polyunsaturated fatty acid biosynthesis pathway. Mar. Drugs 2020, 18, 118. [CrossRef] [PubMed]

35. Damude, H.G.; Zhang, H.; Farrall, L.; Ripp, K.G.; Tomb, J.F.; Hollerbach, D.; Yadav, N.S. Identification of bifunctional $\Delta 12 / \omega 3$ fatty acid desaturases for improving the ratio of $\omega 3$ to $\omega 6$ fatty acids in microbes and plants. Proc. Natl. Acad. Sci. USA 2006, 103, 9446-9451. [CrossRef] [PubMed]

36. Garwin, J.L.; Klages, A.L.; Cronan, J.E., Jr. $\beta$-Ketoacyl-acyl carrier protein synthase II of Escherichia coli. Evidence for function in the thermal regulation of fatty acid synthesis. J. Biol. Chem. 1980, 255, 3263-3265.

37. Kihara, A. Very long-chain fatty acids: Elongation, physiology and related disorders. J. Biochem. 2012, 152, 387-395. [CrossRef]

38. Kim, R.J.; Kim, H.J.; Shim, D.; Suh, M.C. Molecular and biochemical characterizations of the monoacylglycerol lipase gene family of Arabidopsis thaliana. Plant J. 2016, 85, 758-771. [CrossRef]

39. Chattopadhyay, M.K.; Raghu, G.; Sharma, Y.V.R.K.; Biju, A.R.; Rajasekharan, M.V.; Shivaji, S. Increase in oxidative stress at low temperature in an Antarctic bacterium. Curr. Microbiol. 2011, 62, 544-546. [CrossRef]

40. De Maayer, P.; Anderson, D.; Cary, C.; Cowan, D.A. Some like it cold: Understanding the survival strategies of psychrophiles. EMBO Rep. 2014, 15, 508-517. [CrossRef]

41. Paradies, G.; Paradies, V.; De Benedictis, V.; Ruggiero, F.M.; Petrosillo, G. Functional role of cardiolipin in mitochondrial bioenergetics. Biochim. Biophys. Acta 2014, 1837, 408-417. [CrossRef]

42. Demel, R.A.; Kruyff, B. The function of sterols in membranes. Biochim. Biophys. Acta 1976, 457, 109-132. [CrossRef]

43. Hemmi, H.; Julmanop, C.; Hirata, D.; Tsuchiya, E.; Takemoto, J.Y.; Miyakawa, T. The physiological roles of membrane ergosterol as revealed by the phenotypes of syr1/erg3 null mutant of Saccharomyces cerevisiae. Biosci. Biotechnol. Biochem. 1995, 59, 482-486. [CrossRef] [PubMed] 
44. Quilodrán, B.; Hinzpeter, I.; Hormazabal, E.; Quiroz, A.; Shene, C. Docosahexaenoic acid (C22:6n-3, DHA) and astaxanthin production by Thraustochytriidae sp. AS4-A1 a native strain with high similitude to Ulkenia sp.: Evaluation of liquid residues from food industry as nutrient sources. Enzyme Microb. Technol. 2010, 47, 24-30. [CrossRef]

45. Shene, C.; Leyton, A.; Rubilar, M.; Pinelo, M.; Acevedo, F.; Morales, E. Production of lipids and docosahexaenoic acid by a native Thraustochytrium strain. Eur. J. Lipid Sci. Technol. 2013, 115, 890-900. [CrossRef]

46. Grabherr, M.G.; Haas, B.J.; Yassour, M.; Levin, J.Z.; Thompson, D.A.; Amit, I.; Adiconis, X.; Fan, L.; Raychowdhury, R.; Zeng, Q.; et al. Full-length transcriptome assembly from RNA-seq data without a reference genome. Nat. Biotechnol. 2011, 29, 644-652. [CrossRef] [PubMed]

47. Li, B.; Dewey, C.N. RSEM: Accurate transcript quantification from RNA-Seq data with or without a reference genome. BMC Bioinform. 2011, 12, e323. [CrossRef] [PubMed]

48. Robinson, M.D.; McCarthy, D.J.; Smyth, G.K. EdgeR: A bioconductor package for differential expression analysis of digital gene expression data. Bioinformatics 2010, 26, 139-140. [CrossRef] [PubMed]

49. Mi, H.; Muruganujan, A.; Huang, J.X.; Ebert, D.; Mills, C.; Guo, X.; Thomas, P.D. Protocol update for large-scale genome and gene function analysis with the PANTHER classification system (v.14.0). Nat. Protoc. 2019, 14, 703-721. [CrossRef]

50. Young, M.D.; Wakefield, M.J.; Smyth, G.K.; Oshlack, A. Gene ontology analysis for RNA-seq: Accounting for selection bias. Genome Biol. 2010, 11, R14. [CrossRef]

Publisher's Note: MDPI stays neutral with regard to jurisdictional claims in published maps and institutional affiliations.

(C) 2020 by the authors. Licensee MDPI, Basel, Switzerland. This article is an open access article distributed under the terms and conditions of the Creative Commons Attribution (CC BY) license (http://creativecommons.org/licenses/by/4.0/). 